\title{
Deploying hand off mechanism with the software defined network vs mobile IP for 5G network: A feasibility study
}

\author{
Khaled Y Alghamdi ${ }^{1 *}$, Robin Braun ${ }^{2}$ \\ ${ }^{1,2}$ School of Electrical and Data Engineering, University of Technology, Sydney Sydney, Australia
}

\section{Keywords}

Mobile IP

SDN

NFV

Hand off

Received: 11 February 2019

Accepted: 12 March 2019

Published: 20 April 2019

\begin{abstract}
In recent times the Software Defined Network (SDN), Network Functions Virtualization (NFV), Mobile Edge Computing (MEC), and Cloud Radio Access Network (C-RAN) have evolved as emerging technologies with high performance computing capabilities for the deployment of network functions such as mobile Evolved Packet Core (EPC), firewalls, local cache, virtual base station, etc. Also the of the abstractions provided by Software Networks technologies (essentially SDN and NFV) to support an abstracted model for any 5G network function, independent of its nature (network, computational, storage) and the implied resources (optical, wireless, satellite, cloud, etc). The growth in telecommunications has also led to increased deployment of Mobile Internet Protocol (IP) technology besides General Packet Radio Services (GPRS), 3G and 4G Cellular networks for high-end data or packet switched calls. Mobile IP is an outcome of convergence of cellular communication with the IP networks as a Next Generation Network application on traditional networks to support real-time multimedia services, network mobility and 2-way access. However, there are limitations with the Mobile IP which has its own challenges for implementing in the network which includes Security issues like Denial-of-Service attack, Theft of information Passive Eaves Dropping, insider attack, etc. Further, in Mobile IP there exist triangulation problems between the sending and receiving nodes along with latency issues during handoff for the mobile nodes causing huge burden in the network. This paper will try to compare some of the existing deployments of Mobile IP and make comparative analysis with the SDN implementation models for the Cellular Network for the Handoff mechanism.
\end{abstract}

(C) 2019 The Author(s). Published by TAF Publishing.

\section{INTRODUCTION}

As mobile network becomes difficult to scale with growing video traffic and there is an increase dependency on Operations Support System (OSS) system and its experts for these mobile networks and are inflexible and costly. Open Flow as mentioned in $[1,2]$ gives several advantages.

Further as we see the use cases interference mitigation due to inter cell interference in a wireless system are performed in a distributed fashion and with the Coordinated Multipoint (CoMP) techniques extensive computational processing is involved. SDN Controller with Open Flow is implemented in the Central control layer and optimizes the Radio Resource Management (RRM) Module of the Long Term Evolution (LTE) network for resource allocation. Thus, upgrading the RRM is possible with this implementation using the Open flow that is southbound interface as can be seen in the Figure 1.

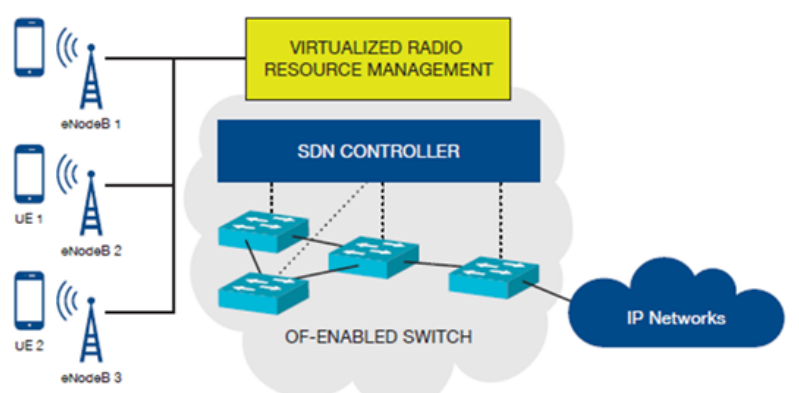

Fig. 1. Open flow enabled network for interference mitigation

This makes SDN Open flow a feasible solution as a replacement for Mobile IP for the HandOff Mechanism. Further in $[3,4]$. Mobile IP network has challenges related to its secu-

\footnotetext{
${ }^{*}$ Corresponding author: Khaled Y Alghamdi

†email: kyadg903@hotmail.com
} 
rity issues where when the mobile registers a new care-ofaddress, of a foreign agent to a home agent a malicious attack can occur after registration with the mobile node. With triangular routing and IP-IP tunneling of the foreign agent and home agent there is a delay and probability of having network congestion. There are handoff issues of mobile devices with the home agent as huge traffic conditions during handoff cannot be handled in this scenario. In this paper in Section II will try to explore on the existing Handoff models for Mobile IP and see how the latency issues are being currently handled, Section III we will also review various Handoff Models for SDN Open Flow in LTE and 5G for Handoff. Further, Section IV we will propose our own SDN Handoff Model with SDN and non-SDN controller network.

\section{HAND OFF MODELS WITH MOBILE IP}

The study of hand off mechanisms in Mobile IP in $[5,4]$ discusses the following Hand off Mechanisms with Mobile IP as follows:

\section{A. Hierarchial Mobile IP(HMIP)}

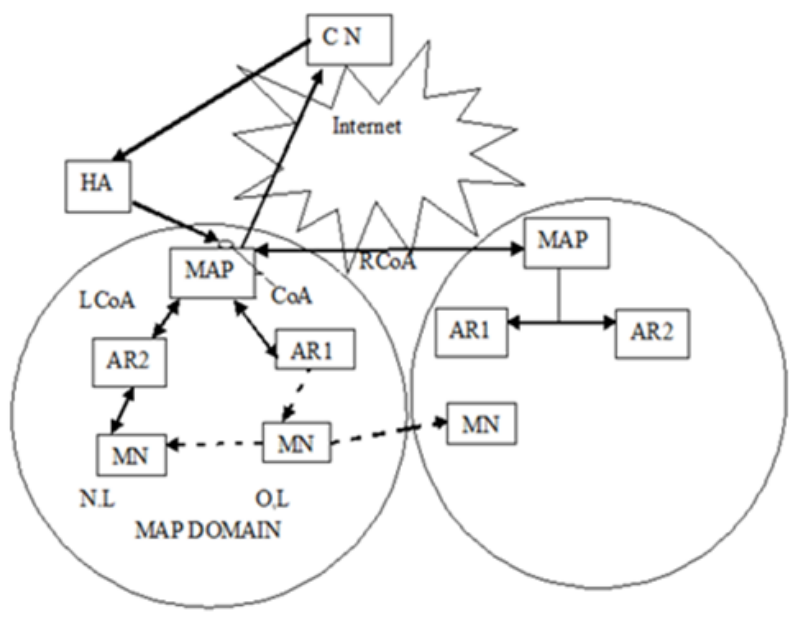

Fig. 2. HMIP

This is one of the enhanced mobile IP schemes for the Mobile IPv6 for handovers and has been developed to resolve registration delays during the HA (Home Agent) registration process and resolution delay when a new CoA (or Careof-Address) is configured for the MN(Mobile Node) in foreign network. Here the MN has micro or macro mobility with the single or different administrative domains respectively. In HMIP as seen in Figure 2 the MAP (Mobile Anchor Points) is the router and is considered as HA of the MN. The MN addresses targeted with packets are intercepted by the MAP inside the domain and are accordingly tunneled with the CoA as correspondent of the MNs in their foreign network.
So, when MN moves inside the domain it registers its CoA to MAP and is not informed to HA and is defined as Local Care of Address (LCoA). When the MN moves outside to a new MAP domain the Regional Care of Address (RCoA) is obtained and a binding update to the MAP is sent by the MN binding the MN's RCoA to its LCoA. MAP then sends binding acknowledgement to $\mathrm{MN}$ for informing successful registration. Another binding update is sent to MN's HA when MN changes the entire MAP domain.

Thus, this network will reduce the overheads due to signaling including the delays in handover reducing the home agent registration when MN moves inside the domain in $[6,7]$ however, it states that for slow moving nodes and global communication this model is not suitable.

\section{B. Fast Handover Mobile IP (FMIP)}

As mentioned in [8] in RFC 5268 following reference scenario may be considered for the FHMIP in Figure 3 as follows:

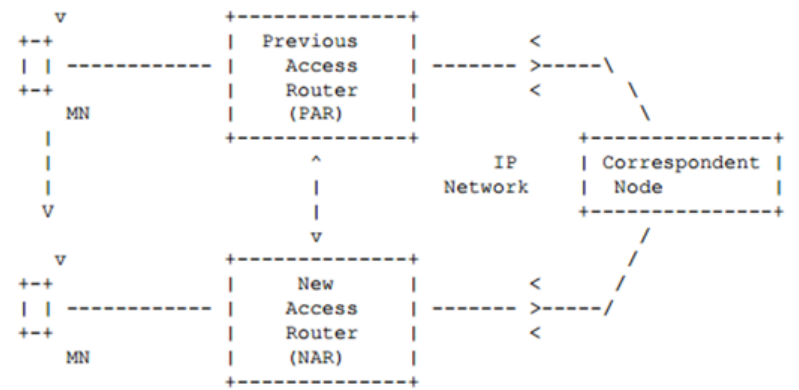

Fig. 3. Reference architecture for FMIP

To address FA address resolution delay, a new CoA is preconfigured with MN as it moves from old Access Router (PAR) to New Access Router (NAR) and uses wireless link layer (L2) trigger-based information for smoothing of handover procedure and minimizing the FA resolution delay. There are 3 types of fast handover MN initiated Handover, Network Initiated Handover and Reactive Handover as mentioned in [3]. However, we do see issues during triangular routing conditions may cause high bandwidth utilization and increased latency even with FMIP.

\section{Mobile IP Issues and Deploying SDN Open Flow with Mobile IP}

In [3] it was observed that Mobile IP suffers from long handover latencies causing packet loss as mobile moves from one domain to the other, there are tunneling overheads, signaling overhead due to multiple registrations, consumes large amount of network performance and the extra binding update creates and overhead. To enable no changes in MN during handover in [9] explores the Proxy Mobile IP (PMIP) 
based network deployment and further discusses the implementation of the Open Flow architecture with PMIP with the reference architecture in Figure 4.

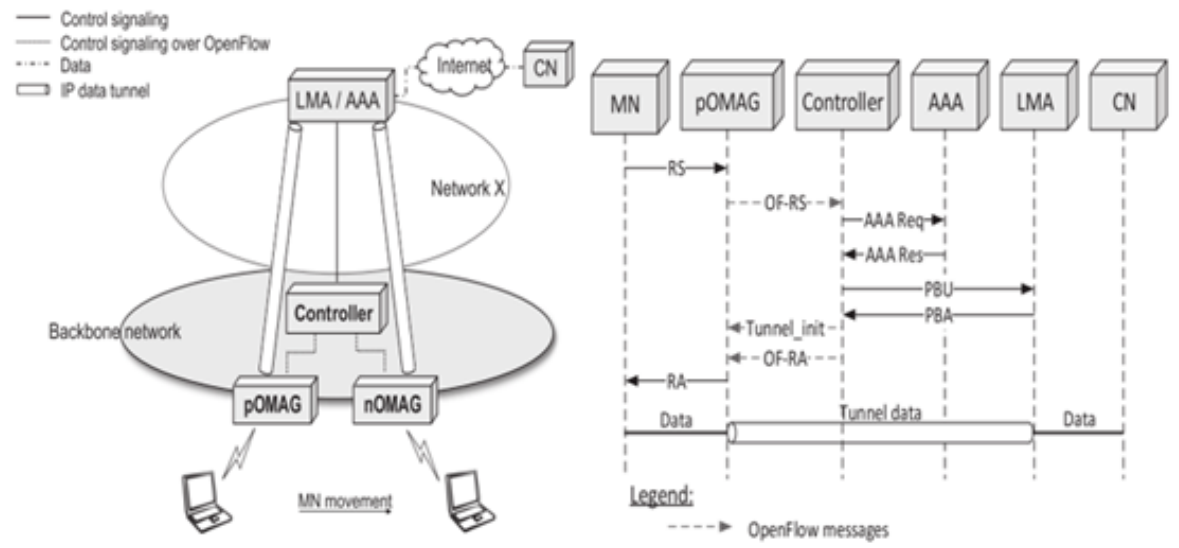

Fig. 4. OF-PMIP architecture and mobile node registration

The controller here performs on behalf of the Mobile Access Gateway (MAG) and communicates with the LMA in the PMIP domain. The OF-PMIP here works either in proactive or reactive mode based on the OMAG configurations for link monitoring and tunnel management for the MN with the network. In the proactive OF-PMIP Open Flow based Proxy Mobile IP the IP tunnel status of MN is maintained by the Open Flow Mobile Access Gateway (OMAG) as temporary or conclusive. In [9] it is further established that proactive OFPMIP indicates lower latency as compared to the PMIP or reactive OF-PMIP and supports seamless mobility and no disruption on real time services giving QoS experience to the mobile users.

\section{HAND OFF MODELS WITH SDN OPEN FLOW}

To be able to ensure reduced latency with Handover of UEs within the network domain with QoE to users as a part of the LTE /LTE Advanced, 5G Networks or Enhanced Mobile Network. We may consider from [3] developing Hand off Models for the mobiles as a part of SDN network. This includes no change with the user session on the UE mobility based on the various SDN setups specified in [10] explained here.

\section{A. Centralized SDN}

We may develop a Centralized SDN Network for inter operator handover and intra frequency or inter frequency handover with reference to the architecture in Figure 5 as follows:

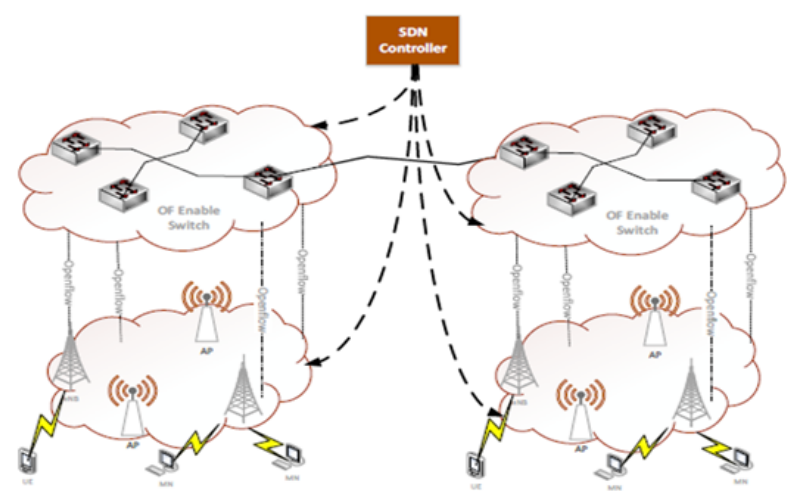

Fig. 5. Centralised SDN network setup for handoff

In the case of evolved LTE and 5G network MME, PCRF and Target eNodeBs and Source eNodeBs the handover functionality is being handled by the centralized SDN controller. The advantage to deploy this solution there is no specific implementation or support related to Mobile Node and the IP address remains unchanged. This also reduces the round trip time between the eNodeBs, MMEs and the Mobile Nodes. With a proactive setup using Open Flow the latency time can be further reduced. Here using the RRM messages on the SDN controller mobile SDN is also enabled 
Disadvantage of deploying another SDN network with a centralized SDN controller will require implementation as data centric and highly compute intensive considering inter operator handover scenarios making it cost intensive.

\section{B. Deploying Multiple SDN Controller Network}

The Multi SDN Controller Network can be set as follows:

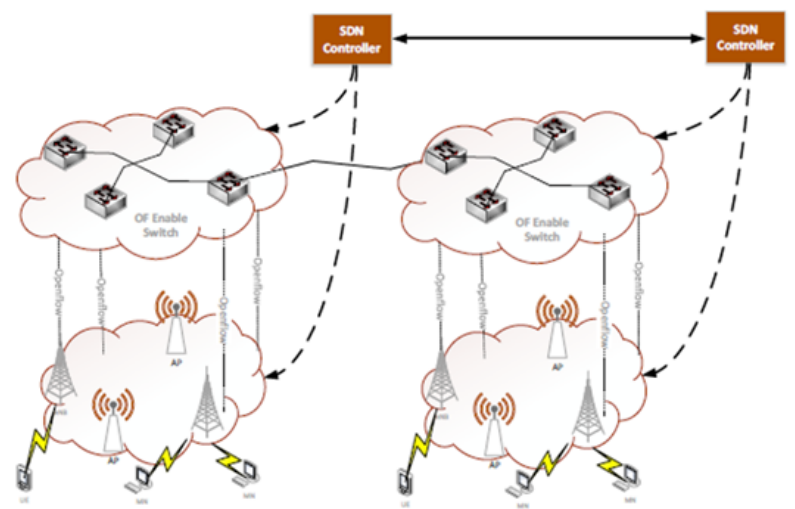

Fig. 6. Geographically separated SDN controllers for multi SDN controller handover mechanism

1) Semi centralized SDN controller: Here the network will be separated into different domains within the same setup or geographically separated setup. Each SDN domain will function as part of the same SDN domain. During the handover, mobile will need to inform about the SDN domain it intends to attach with and update its status for continued user sessions. The SDN cloud can also be deployed using for each of these SDN domains to handle seamless handover based on traffic condition with the mobile SDNs.

2) Hierarchial SDN controllers: We may consider the Hierarchial Cell Structure as mentioned in [11]:

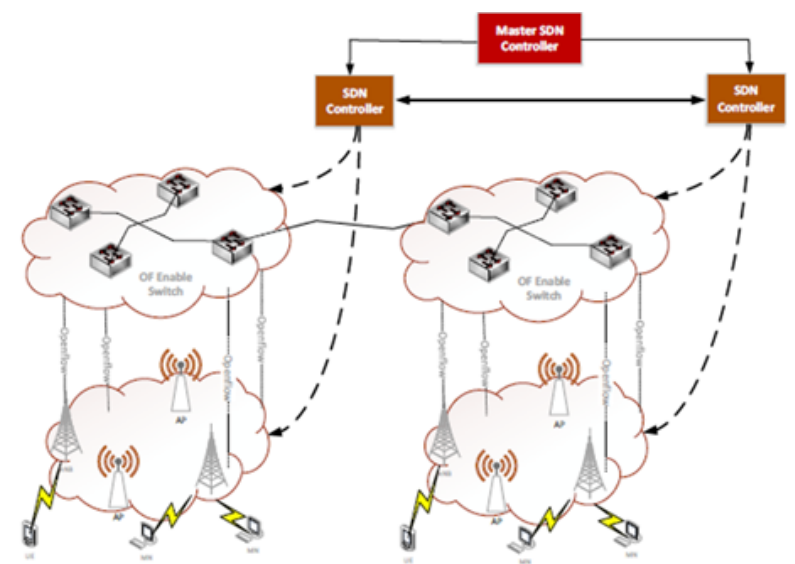

Fig. 7. Handoff with hierarchical SDN controller setup

It is seen in Figure 7 that with the network control plane for different Functional Elements distributed as higher or lower layer the current setup will not only manage the control plane functionality for handover but also enable sup- port for power control and other interference mitigation. However, it is expected that the information exchange will be highly complex in nature and will include huge implementation and maintenance efforts. It is analyzed that an SDN Cloud setup is highly recommended to reduce the computational complexity and to simplify the exchange of these messages across the hierarchy.

\section{PROPOSING HAND OFF MECHANISM IN SDN FOR 5G NETWORK}

The author based on the analysis done in Section III propose following Hand off Mechanism Model for a 5G Network with advantages of SDN over Mobile IP.

\section{A. Proposing SDN Controllers for 5G Network Slicing}

Another model can be developed for Network Slicing by deploying SDN on it as mentioned in [12] for the 5G Network in Figure 7. The authors found that this can be extended for the Handoff mechanism based on the setup from the SDN model of Figure 6.

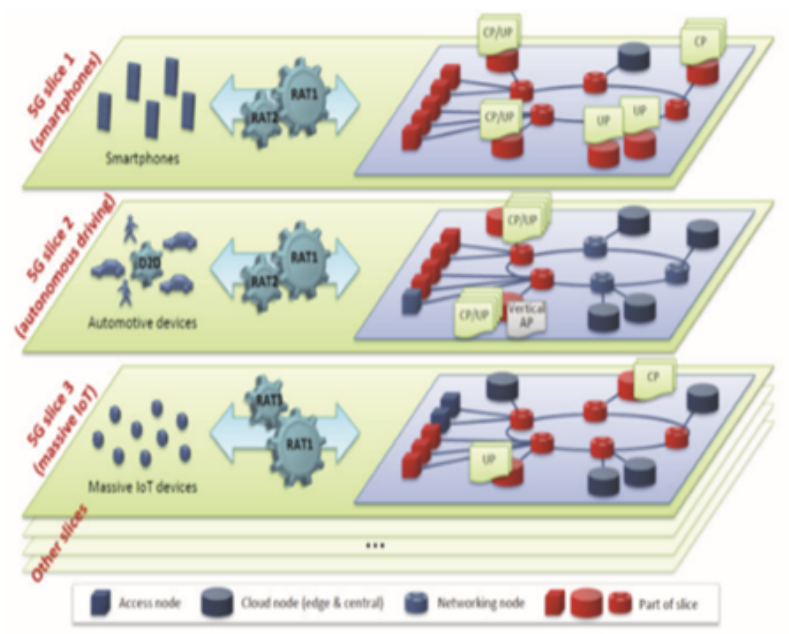

Fig. 8. Proposing SDN controller for network slicing implementation

This also mentions fulfillment of requirement regarding the sharing of RAN resources among different slices, it is required to efficiently use radio and transport network resources between different slices (including both common channels and dedicated channels). From that perspective some radio Network Functions (such as schedulers) are either defined as "common NFs" or "independent/dedicated NFs" with (at least some) coordination mechanisms between NFs from different slices. This means that the radio NFs of one slice do not operate in complete isolation from another slice. Possible implementation of such scenario could include a set of common radio NFs which provides slicing as a service to higher layers for which North- 
bound APIs can be used for deploying SDN Controllers from the SDN Reference Architecture in [13]. The admission control mechanisms can be protected deploying SDN interfaces for different slices so that congestion in the channel of one slice does not have impact on the other slice with seamless handoff. Further, Priority Scheduling mechanism can be deployed for call congestion scenarios while dealing Call capacity handing in SDN Radio Network Slicing configurations with constrained radio resources allocated during the handoff. The priority scheduling criteria can be implemented with parameters like "Timing Advance" sent in the measurement reports by the UE to its Radio Resource Units.

\section{B. Hand Off Model from SDN to non-SDN Network}

In the feasibility study carried out by the author it was felt that for SDN Handoff implementation we are using Abstraction of Physical Infrastructure via Southbound API from the SDN Reference Architecture in [13]. Further, implementing the SDN Network solutions with existing networks which are not supporting SDN Open Flow can be compute intensive The author felt the need to create interworking for different Handoff Scenarios for the SDN Cellular Network Architecture from [3] with the existing 4G Networks (LTE, LTE Advanced and WiFi) and extending to 3G. It was felt to deploy seamless handover of UE between SDN and non- SDN Domain can also be deployed using the topology in Figure 8 that has been proposed based on SDN Controller to Controller Communication Model as mentioned in [13].

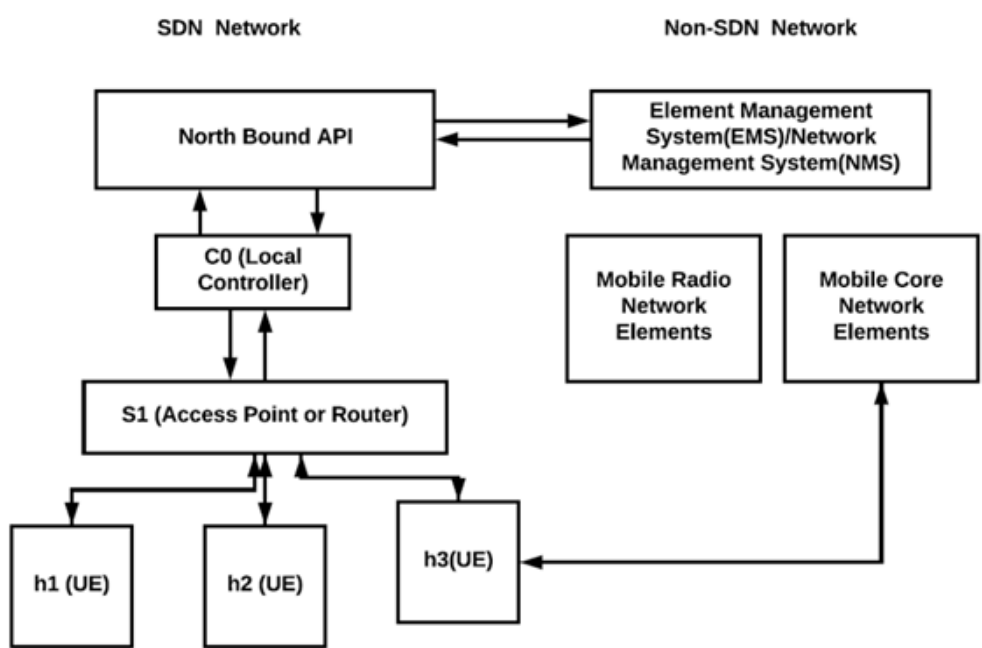

Fig. 9. Topology for hand off mechanism for a non-SDN to SDN network using a wrapper between EMS/NMS and the Northbound API of the SDN network

Here the mobility of the Host 3 (UE) moves from a non-SDN network (i.e, a GSM or LTE or 3G Network without Open Flow) to an SDN Network. The author further proposes an algorithm for the wrapper in this case in the North bound API with the EMS/NMS as:

TABLE 1

ALGORITHM

\begin{tabular}{l}
\hline Handoff Wrapper with Non-SDN Network \\
1: Set up a Border Gateway Protocol between the SDN and non-SDN Network at the control plane \\
2: Setup a TCP session between Northbound API and the API on the EMS/NMS port of the non-SDN Network \\
3: Setup S1 as a mobility switch \\
4: As the host h3 leaves the source non-SDN network send the QoS profile via EMS/NMS API to \\
the North Bound API of the target SDN network and also send the allocated IP address \\
5: Attach host h3 to the Switch S1
\end{tabular}

\section{CONCLUSION}

During the feasibility study on various models studied by the author they were able to develop proposed topology and models for Handoff mechanism with SDN Open Flow making it the most feasible solution for its deployment in the 5G Network. It was found that since SDN does not deploy tri- 
angular routing seamless handover latency issue is lower as compared to Mobile IP which will need higher bandwidth. This was identified in the case when no change was seen in the user session as it moves from source to a target network in a Mobile IP environment even in a 5G Network with FMIP and HMIP implementation for Mobile IP. The author further summarized that the capacity constraint may lead to call congestion for the SDN network slicing. The author proposes priority scheduling using "Timing Advance" pa- rameters sent as a part of measurement report by UEs to the Radio Resource Unit and can be taken as future scope of work for understanding its implementation. This should also take care of latency issues for mobiles at varying distances and handled by multiple SDN controllers. The author, also intends to verify the SDN deployments in a simulated environment and consider verifying the interworking of the SDN with the existing non-SDN Network for handoff and verify security issues during handoff.

\section{REFERENCES}

[1] C. Kolias, S. Ahlawat, C. Ashton, M. Cohn, S. Manning, and S. Nathan, "Openflow-enabled mobile and wireless networks," Open Network Foundation, vol. 2, no. 1, pp. 1-13, 2013.

[2] S. H. Al Ahmadi, "Survey of designing user interface for mobile applications," Journal of Advances in Technology and Engineering Studies, vol. 3, no. 2, pp. 74-79, 2017. doi: https://doi.org/10.20474/jater-3.2.4

[3] R. Ramakrishnaiah, A. A. Alkheraif, D. D. Divakar, K. F. Alghamdi, J. P. Matinlinna, C. Y. K. Lung, S. Cherian, and P. K. Vallittu, "The effect of lithium disilicate ceramic surface neutralization on wettability of silane coupling agents and adhesive resin cements," Silicon, vol. 10, no. 6, pp. 2391-2397, 2018. doi: https://doi.org/10.1007/s12633-017-9670-6

[4] O. A. Osahenvemwen and O. F. Odiase, "Effective management of handover process in mobile communication network," Journal of Advances in Technology and Engineering Studies, vol. 2, no. 6, pp. 176-182, 2016. doi: https://doi.org/10. 20474/jater-2.6.1

[5] G. Chellani and A. Kalla, "A review: Study of handover performance in mobile IP," International Journal of Computer Networks \& Communications, vol. 5, no. 6, pp. 137-151, 2013. doi: https://doi.org/10.5121/ijcnc.2013.5608

[6] A. H. Hashim, F. Anwar, S. Mohd, and H. Liyakthalikh, "Mobility issues in hierarchical mobile IP," in IEEE 3rd International Conference SETIT, New York, NY, 2005.

[7] R. Adamu, "A usability evaluation of mobile banking applications in Nigeria,"' International Journal of Technology and Engineering Studies, vol. 3, no. 1, pp. 38-44, 2017. doi: https://doi.org/10.20469/ijtes.3.40005-1

[8] R. Koodli, "System and method for providing address discovery of services in mobile networks," 2003. [Online]. Available: https://bit.ly/2QmT5lV

[9] S. M. Raza, D. S. Kim, D. Shin, and H. Choo, "Leveraging proxy mobile IPV6 with SDN," Journal of Communications and Networks, vol. 18, no. 3, pp. 460-475, 2016. doi: https://doi.org/10.1109/jcn.2016.000061

[10] S. Kukliński, Y. Li, and K. T. Dinh, "Handover management in SDN-based mobile networks,' in IEEE Globecom Workshops (GC Wkshps), Seoul, South Korea, 2014.

[11] Network, Evolved Universal Terrestrial Radio Access, “3rd generation partnership project: Technical specification group services and system aspects General Packet Radio Service (GPRS) enhancements for evolved universal terrestrial radio access network," 2011. [Online]. Available: https://bit.ly/2ZOQGUd

[12] V. Garcia Cruz, "5g radio access network slicing," B.S. thesis, Universitat Politècnica de Catalunya, Barcelona, Spain, 2018.

[13] J. Ordonez-Lucena, P. Ameigeiras, D. Lopez, J. J. Ramos-Munoz, J. Lorca, and J. Folgueira, “Network slicing for 5G with SDN/NFV: Concepts, architectures, and challenges," IEEE Communications Magazine, vol. 55, no. 5, pp. 80-87, 2017. doi: https://doi.org/10.1109/mcom.2017.1600935 